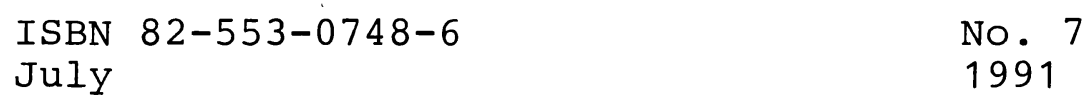

Second order asymptotics for the number of times an estimator is more than $\varepsilon$ from its target value

by

Nils Lid Hjort ${ }^{a, b}$

and

Grete Fenstad ${ }^{a}$ 


\title{
Second order asymptotics for the number of times an estimator is more than $\varepsilon$ from its target value
}

\author{
Nils Lid Hjort ${ }^{a, b}$ and Grete Fenstad ${ }^{a}$ \\ University of Oslo ${ }^{a}$ and Norwegian Computing Centre ${ }^{b}$ \\ -- July 1991 --
}

Abstract. Suppose $\left\{\hat{\theta}_{n}: n \geq 1\right\}$ is a strongly consistent sequence of estimators for a parameter $\theta$, where $\widehat{\theta}_{n}$ is based on the first $n$ observations. Consider $Q_{\varepsilon}$, the number of times $\left|\widehat{\theta}_{n}-\theta\right| \geq \varepsilon$. In another paper (Hjort and Fenstad, 1991) we have shown that $\varepsilon^{2} Q_{\varepsilon}$ has a limit distribution as $\varepsilon \rightarrow 0$, depending only on $\sigma$, the standard deviation of the limit distribution for $\sqrt{n}\left(\widehat{\theta}_{n}-\theta\right)$, under natural regularity conditions. The present paper investigates some second order asymptotics for differences between $Q_{\varepsilon}$-variables. The limit of $E\left(Q_{\varepsilon, 1}-Q_{\varepsilon, 2}\right)$ is calculated in cases where $E Q_{\varepsilon, 1} / E Q_{\varepsilon, 2}$ goes to 1, and this is used to distinguish between competing estimators with identical limit distributions. Thus using denominator $n-\frac{1}{3}$ in the familiar formula for estimating a normal variance is better than both $n$ and $n-1$ and indeed all other choices, for example, in the sense of leading to the smallest possible expected number of $\varepsilon$-errors. Results of this type are found in a selection of familiar estimation problems, using limit results for expected differences. Some second order distributional results are reached as well. It is shown how $\varepsilon$ times a $Q_{\varepsilon}$-difference tends to a variable which is related to some exponential distributions associated with Brownian motion, and that have recently been investigated by Hjort (1991).

KEY WORDS: asymptotic relative deficiency, asymptotic relative efficiency, comparison of estimators, loss function, second order optimality, total relative time for Brownian motion, the number of $\varepsilon$-misses

1. Introduction. Suppose $X_{1}, X_{2}, \ldots$ is a sequence of independent observations from some distribution $F$, and let $\theta$ be some parameter of interest. Assume that $\widehat{\theta}_{n}$ is based on the first $n$ data points, and that this estimator sequence is strongly consistent, i.e. $\widehat{\theta}_{n} \rightarrow \theta$ almost surely. Then $Q_{\varepsilon}$, the number of cases where $\left|\widehat{\theta}_{n}-\theta\right| \geq \varepsilon$, is finite almost surely. Under natural regularity conditions, which include existence of a normal $\left(0, \sigma^{2}\right)$ limit for $\sqrt{n}\left(\widehat{\theta}_{n}-\theta\right)$, Hjort and Fenstad (1991, Section 7) have shown that

$$
\varepsilon^{2} Q_{\varepsilon} \rightarrow_{d} Q=\operatorname{Leb}\{s \geq 0:|W(s)| \geq s / \sigma\}=\int_{0}^{\infty} I\{|W(s)| \geq s / \sigma\} \mathrm{d} s,
$$

in which Leb is Lebegue measure on the halfline, $W($.$) is a Brownian motion process, and$ $I\{\ldots\}$ denotes an indicator function. They also show that $\varepsilon^{2} E Q_{\varepsilon} \rightarrow E Q$, which simply is equal to $\sigma^{2}$. Suppose $\sqrt{n}\left(\widehat{\theta}_{n, j}-\theta\right) \rightarrow_{d} N\left\{0, \sigma_{j}^{2}\right\}$ for two competing estimator sequences, and let $Q_{j, \varepsilon}$ be their accompanying number of $\varepsilon$-misses variables. Then a natural asymptotic measure of relative efficiency is

$$
\text { a.r.e. }=\lim _{\varepsilon \rightarrow 0} \frac{E Q_{1, \varepsilon}}{E Q_{2, \varepsilon}}=\frac{\sigma_{1}^{2}}{\sigma_{2}^{2}}
$$


This is also the traditional formula for a.r.e. based on calculations of asymptotic ratios $n_{1} / n_{2}$ in which $n_{1}$ and $n_{2}$ are the sample sizes needed by methods 1 and 2 to achieve the same level of mean squared error precision. Hjort and Fenstad (1991) also give generalisations of (1.1) and (1.2) to multi-dimensional parameters and general distance measures, and reach limit results for $Q_{\varepsilon}$-variables in more involved problems like nonparametric estimation of distribution functions and densities.

The $Q_{\varepsilon}$-based criterion (1.2) is only a 'first order measure' and cannot distinguish between estimator sequences with identical limit distributions, in which case typically $\varepsilon^{2}\left\{Q_{1, \varepsilon}-Q_{2, \varepsilon}\right\} \rightarrow 0$ and $Q_{1, \varepsilon} / Q_{2, \varepsilon} \rightarrow 1$ in probability. Our aim in this paper is to develop some 'second order' theory for differences of $Q_{\varepsilon}$ 's, which then can be applied to single out the 'best' estimator in a class of estimators with the same limit distribution. Being 'best' here means having the smallest possible expected number of $\varepsilon$-errors, in the limit as $\varepsilon$ goes to zero. In particular we intend to solve comparison problems for unbiased minimum variance (UMV) estimators versus maximum likelihood (ML) estimators versus Bayes estimators in some familar cases where they disagree. This is in a spirit similar to Hodges and Lehmann (1970) who worked with a measure of asymptotic relative deficiency (a.r.d.), expressed as the limit of sample size difference $n_{1}-n_{2}$ in cases where $n_{1} / n_{2} \rightarrow 1$; see also Lehmann (1983, Chapter 5.2).

In Section 2 formulae are found for a $Q_{\varepsilon}$-based

$$
\text { a.r.d. }=\lim _{\varepsilon \rightarrow 0} E\left\{Q_{1, \varepsilon}-Q_{2, \varepsilon}\right\}
$$

in the simple case of estimating a mean parameter, using estimators of the Bayesian variety $\frac{n}{n+c} \bar{X}_{n}+\frac{c}{n+c} d$. In this a.r.d. formula the underlying skewness of the $X_{i}$ 's enters in a natural way, whereas it does not in Hodges and Lehmann's calculations. The a.r.d. formula makes it possible to show that $n-\frac{1}{3}$ is the superior choice of denominator in the familiar $\sum_{i=1}^{n}\left(X_{i}-\bar{X}_{n}\right)^{2} /(n+c)$ formula for a normal variance, for example. This and other mean-parameter related examples are treated in Section 3. A couple of technically more demanding problems are included in Section 4 . We are able to show that $\left(\bar{X}_{n}\right)^{2}+\widehat{\sigma}_{n}^{2} / n$, as an estimator for the squared normal mean, can be expected to exhibit fewer $\varepsilon$-errors than all other estimators of the form $\left(\bar{X}_{n}\right)^{2}+d \widehat{\sigma}_{n}^{2} / n$, for example.

Comparing estimation methods in terms of $E Q_{\varepsilon}$ can be phrased in decision theoretic terms, where the underlying loss function is equal to the number of $\varepsilon$-errors for the full sequence of estimates. This point is briefly discussed in Section 5, along with some Bayesian considerations.

The theory and applications presented in Sections 2-5 are only concerned with expected values of $Q_{e}$-variables. This is reflected in the tools used, namely Edgeworth expansions and further Taylor type approximations to probabilities. Section 6 presents an informative addendum and is concerned with second order distributional aspects of $Q_{1, \varepsilon}-Q_{2, \varepsilon}$ differences. In the structurally simplest case of estimating a mean parameter it turns out that

$$
\varepsilon^{2}\left\{Q_{1, \varepsilon}-Q_{2, \varepsilon}\right\} \rightarrow_{p} 0 \text { and } Q_{\varepsilon, 1} / Q_{\varepsilon, 2} \rightarrow_{p} 1 \text { while } \varepsilon\left\{Q_{1, \varepsilon}-Q_{2, \varepsilon}\right\} \rightarrow_{d} A-B
$$


say, where $A$ and $B$ are certain total relative time variables associated with Brownian motion, with distributions that are exponential or mixtures of exponentials and unit point masses at zero; see Hjort (1991) for a separate account on these.

Our paper is mainly reporting on an investigation into one particular way of distinguishing between first order equivalent sequences. We do not claim that the a.r.d. criterion (1.3) on which our second order comparisons are based is statistically more natural than other criteria, but it is interesting that it can be computed at all, and to see how it fares compared to other second order criteria, of which several others exist. In addition to the a.r.d.-calculations of Hodges and Lehmann and ours of the present paper, Rao (1962) and others have worked with asymptotic sufficiency, Pfanzagl (1973) and others with coverage probabilities and median unbiasedness, and Ghosh and Subramanyam (1974) and others with expansions for mean squared errors. A good source for further information is the discussion of the paper by Berkson (1980). Let us finally point out that techniques of the present paper can be used to establish expansion results for coverage probabilities of the type $p_{n}=\operatorname{Pr}\left\{\left|\widehat{\theta}_{n}-\theta\right| \leq k / \sqrt{n}\right\}$. These results, which in a way are simpler than those of Sections 2-5, could then be used to compare different estimators with the same limit distribution. Such comparisons would however depend on the value of $k$.

2. General results for estimating a mean. Suppose $X_{1}, X_{2}, X_{3}, \ldots$ are i.i.d. with $E X_{i}=\xi, \operatorname{Var} X_{i}=\sigma^{2}$, and skewness $E\left(X_{i}-\xi\right)^{3} / \sigma^{3}=\gamma$. Our first aim in this section is to prove the following.

Proposition 1. Let $a$ be positive, and consider $Q_{\varepsilon}(c)$, the number of times, among $n \geq a / \varepsilon^{2}$, where $\left|\frac{n}{n+c} \bar{X}_{n}-\xi\right| \geq \varepsilon$. Suppose that $X_{i}$ has finite fourth order moment and that its distribution is non-lattice, and write $\phi($.$) for the standard normal density. Then$

$$
\begin{aligned}
\lambda_{a}(c) & =\lim _{\varepsilon \rightarrow 0} E\left\{Q_{\varepsilon}(c)-Q_{\varepsilon}(0)\right\} \\
& =2\left[\frac{\xi^{2}}{\sigma^{2}} c^{2}-\left(2-\frac{2 \gamma}{3} \frac{\xi}{\sigma}\right) c\right] \int_{\sqrt{a} / \sigma}^{\infty} \phi(y) \mathrm{d} y-\frac{2 \gamma}{3} \frac{\xi}{\sigma} \frac{\sqrt{a}}{\sigma} \phi(\sqrt{a} / \sigma) c
\end{aligned}
$$

ProOF: We choose to work with $Q_{\varepsilon}(c)$ in the form

$$
Q_{\varepsilon}(c)=\sum_{n \geq a m} I\left\{\left|\frac{n}{n+c} \bar{X}_{n}-\xi\right| \geq \frac{1}{\sqrt{m}}\right\}
$$

writing $m=1 / \varepsilon^{2}$. Note that $Q_{\varepsilon}(c)$ is finite a.s. by the strong law of large numbers. Consider $T_{n}=\sqrt{n}\left(\bar{X}_{n}-\xi\right) / \sigma$. Inserting $\bar{X}_{n}=\xi+\sigma T_{n} / \sqrt{n}$ the indicator function term in (2.2) can be written $I\left\{T_{n} \leq l(c)\right.$ or $\left.T_{n} \geq r(c)\right\}$, where

$$
l(c)=-\frac{1}{\sigma} \sqrt{\frac{n}{m}}+\frac{1}{\sqrt{n}} \frac{c \xi}{\sigma}-\frac{1}{\sqrt{n m}} \frac{c}{\sigma}, \quad r(c)=\frac{1}{\sigma} \sqrt{\frac{n}{m}}+\frac{1}{\sqrt{n}} \frac{c \xi}{\sigma}+\frac{1}{\sqrt{n m}} \frac{c}{\sigma} .
$$

Letting $s=n / m$ we have

$$
l(0)=-u, \quad l(c)=-u+a / \sqrt{n}-b / n, \quad r(0)=u, \quad r(c)=u+a / \sqrt{n}+b / n,
$$


in which $u=\sqrt{s} / \sigma, a=c \xi / \sigma$, and $b=c \sqrt{s} / \sigma$. Since

$$
Q_{\varepsilon}(c)-Q_{\varepsilon}(0)=\sum_{n \geq a m}\left[I\left\{T_{n} \leq l(c) \text { or } T_{n} \geq r(c)\right\}-I\left\{T_{n} \leq l(0) \text { or } T_{n} \geq r(0)\right\}\right]
$$

its expected value can be written

$$
E\left\{Q_{\varepsilon}(c)-Q_{\varepsilon}(0)\right\}=\sum_{n \geq a m}\left[\left\{G_{n}(l(c))-G_{n}(l(0))\right\}-\left\{G_{n}(r(c))-G_{n}(r(0))\right\}\right],
$$

in terms of the cumulative distribution function $G_{n}$ of $T_{n}$.

Under the non-lattice assumption there is a Cramér-Edgeworth expansion for $G_{n}$ of the form

$$
G_{n}(t)=\Phi(t)-\frac{1}{6} \frac{\gamma}{\sqrt{n}} A(t) \phi(t)+\frac{1}{n} R(t) \phi(t)+O\left(n^{-3 / 2}\right),
$$

where $A(t)=t^{2}-1$ and $R(t)$ is a certain polynomial of degree five, see for example Barndorff-Nielsen and Cox (1989, Ch. 4). In view of (2.5) we need to approximate terms of the type $G_{n}(t+\delta)-G_{n}(t)$ where $\delta$ is of the order of $1 / \sqrt{n}$. Using Taylor expansions one finds that

$$
G_{n}(t+\delta)-G_{n}(t)=\phi(t) \delta-\frac{1}{2} t \phi(t) \delta^{2}+\frac{1}{6} \frac{\gamma}{\sqrt{n}} B(t) \phi(t) \delta+O\left(n^{-3 / 2}\right),
$$

in which $B(t) \phi(t)$ is the derivative of $-A(t) \Phi(t)$, i.e. $B(t)=t^{3}-3 t$, and the $O$-term is uniform in $t$. This leads to

$$
\begin{aligned}
G_{n}(-u+a / \sqrt{n}-b / n)-G_{n}(-u) & =\phi(u) a / \sqrt{n}+\phi(u)\left\{-b+\frac{1}{2} u a^{2}-\frac{1}{6} \gamma a B(u)\right\} / n \\
G_{n}(u+a / \sqrt{n}+b / n)-G_{n}(u) & =\phi(u) a / \sqrt{n}+\phi(u)\left\{b-\frac{1}{2} u a^{2}+\frac{1}{6} \gamma a B(u)\right\} / n
\end{aligned}
$$

The summand in (2.5) can accordingly be expressed, apart from $O\left(n^{-3 / 2}\right)$ terms, as

$$
\frac{1}{n} \phi(u)\left\{-2 b+u a^{2}-\frac{1}{3} \gamma a B(u)\right\}=\frac{1}{m} \phi\left(\frac{\sqrt{s}}{\sigma}\right) \frac{1}{s}\left\{-2 \frac{\sqrt{s}}{\sigma} c+\frac{\xi^{2} \sqrt{s}}{\sigma^{3}} c^{2}-\frac{\gamma}{3} \frac{\xi}{\sigma} B\left(\frac{\sqrt{s}}{\sigma}\right) c\right\} .
$$

Since $\frac{1}{m} \sum_{n / m \geq a} h(n / m)$ is a Riemannian approximation to the integral $\int_{a}^{\infty} h(s) \mathrm{d} s$, and converges to this limit as $m \rightarrow \infty$, we find in the end that $E\left\{Q_{\varepsilon}(c)-Q_{\varepsilon}(0)\right\}$ converges to

$$
\begin{aligned}
\lambda_{a}(c) & =\int_{a}^{\infty}\left[\frac{\xi^{2}}{\sigma^{3}} \frac{1}{\sqrt{s}} c^{2}-\frac{2}{\sigma} \frac{1}{\sqrt{s}} c-\frac{\gamma}{3} \frac{\xi}{\sigma} \frac{1}{s} B\left(\frac{\sqrt{s}}{\sigma}\right) c\right] \phi\left(\frac{\sqrt{s}}{\sigma}\right) \mathrm{d} s \\
& =2 \int_{\sqrt{a} / \sigma}^{\infty}\left[\frac{\xi^{2}}{\sigma^{2}} c^{2}-2 c-\frac{\gamma}{3} \frac{\xi}{\sigma} \frac{B(u)}{u} c\right] \phi(u) \mathrm{d} u .
\end{aligned}
$$

Some further analysis, using $B(u) / u=u^{2}-3$, finally proves (2.1).

In the applications presented below we will study this and similar limits as functions of $c$, to find the estimator sequence that can be expected to make fewest $\varepsilon$-errors. The optimal value of $c$ as computed from (2.1) will depend upon the somewhat arbitrary value of $a$, however. Write in general $Q_{\varepsilon, m}(c)$ for the number of $\varepsilon$-errors committed by $\frac{n}{n+c} \bar{X}_{n}$ 
among $n \geq m$ cases. The limit result $(2.1)$ for $\lambda_{a}(c)$ relates to $Q_{\varepsilon, a / \varepsilon^{2}}(c)$, and it is natural to let $a$ tend to zero. The limit function $\lambda_{0}(c)$ obtained by letting $a \rightarrow 0$ in (2.1) is not quite the limit of the expected difference between $Q_{\varepsilon, 1}(c)$ and $Q_{\varepsilon, 1}(0)$, since the remainder term $O\left(\sum_{n \geq a m} n^{-3 / 2}\right)$ does not go to zero for $a=1 / m$. The following holds, however.

Proposition 2. Let $Q_{\varepsilon, a(\varepsilon) / \varepsilon^{2}}(c)$ be the number of $\varepsilon$-misses for $\frac{n}{n+c} \bar{X}_{n}$, counted among $n \geq a(\varepsilon) / \varepsilon^{2}$, where $a(\varepsilon) \rightarrow 0$ while $a(\varepsilon) / \varepsilon^{2} \rightarrow \infty$ (as with $a(\varepsilon)=\varepsilon$, for example). Then

$$
\lambda_{0}(c)=\lim _{\varepsilon \rightarrow 0} E\left\{Q_{\varepsilon, a(\varepsilon) / \varepsilon^{2}}(c)-Q_{\varepsilon, a(\varepsilon) / \varepsilon^{2}}(0)\right\}=\frac{\xi^{2}}{\sigma^{2}} c^{2}-2\left(1-\frac{\gamma}{3} \frac{\xi}{\sigma}\right) c .
$$

Sometimes it is reasonable to balance the objective $\bar{X}_{n}$ against some prior value. Bayesians often use estimators of the type $\widehat{\xi}_{n}(c, d)=\frac{n}{n+c} \bar{X}_{n}+\frac{c}{n+c} \xi_{0}$, see Section 5 . Consider therefore the slight extension

$$
Q_{\varepsilon}(c, d)=\sum_{n \geq a / \varepsilon^{2}} I\left\{\left|\frac{n}{n+c} \bar{X}_{n}+\frac{c}{n+c} d-\xi\right| \geq \varepsilon\right\} .
$$

The appropriate generalisation of $(2.3)$ is found to be

$$
l(c, d)=-\frac{1}{\sigma} \sqrt{\frac{n}{m}}+\frac{1}{\sqrt{n}} \frac{c(\xi-d)}{\sigma}-\frac{1}{\sqrt{n m}} \frac{c}{\sigma}, \quad r(c)=\frac{1}{\sigma} \sqrt{\frac{n}{m}}+\frac{1}{\sqrt{n}} \frac{c(\xi-d)}{\sigma}+\frac{1}{\sqrt{n m}} \frac{c}{\sigma},
$$

and by repeating previous arguments mutatis mutandis one proves the following generalisation of (2.7).

Proposition 3. Let as above $\varepsilon$ and $a(\varepsilon)=\varepsilon$ tend to zero. Then

$$
\lambda_{0}(c, d)=\lim _{\varepsilon \rightarrow 0} E\left\{Q_{\varepsilon}(c, d)-Q_{\varepsilon}(0,0)\right\}=\frac{(\xi-d)^{2}}{\sigma^{2}} c^{2}-2\left(1-\frac{\gamma}{3} \frac{\xi-d}{\sigma}\right) c .
$$

A further generalisation of (2.7) and (2.8) is given at the end of $4 \mathrm{C}$ below. We think of these as a.r.d.-formulae, see (1.3) and the remarks made there. We also remark that an alternative way of proving (2.1), (2.7), (2.8) is outlined in Section 6.

REMARK. One may also compute the Hodges and Lehmann asymptotic relative deficiency in this situation, defined as the limit of $n_{0}(c, d)-n_{0}$, where $n_{0}(c, d)$ is the sample size needed to achieve $E\left\{\widehat{\xi}_{n}(c, d)-\xi\right\}^{2}=E\left\{\widehat{\xi}_{n_{0}}(0,0)-\xi\right\}^{2}$, say; see Lehmann (1983, Section $5.2)$ for discussion. Then $n_{0}(c, d) / n_{0}(0,0) \rightarrow 1$ for all $(c, d)$, but one can prove

$$
\text { a.r.d.hl }(c, d)=\lim _{n_{0} \rightarrow \infty}\left\{n_{0}(c, d)-n_{0}(0,0)\right\}=\frac{(\xi-d)^{2}}{\sigma^{2}} c^{2}-2 c .
$$

The a.r.d. formula (2.8) differs from this in that the skewness $\gamma$ also enters, in a natural way.

\section{Some applications.}

3A. Normal mean. Let $X_{i}$ be normal $(\theta, 1)$. Consider the estimator sequence $\widehat{\theta}_{n}(c, d)=$ $\frac{n}{n+c} \bar{X}_{n}+\frac{c}{n+c} d$, and let $Q_{\varepsilon}(c, d)$ be the number $\varepsilon$-misses. This fits into the general scheme 
of Section 2 with $\xi=\theta, \sigma=1$, and $\gamma=0$. Hence

$$
\lambda_{0}(c, d)=\lim _{\varepsilon \rightarrow 0} E_{\theta}\left\{Q_{\varepsilon}(c, d)-Q_{\varepsilon}(0,0)\right\}=(\theta-d)^{2} c^{2}-2 c
$$

If this is averaged over $\theta$ w.r.t. some distribution with 'prior mean' $E \theta=\theta_{0}$ and 'prior variance' $\operatorname{Var} \theta=\tau^{2}$, then one finds $\left\{\tau^{2}+\left(\theta_{0}-d\right)^{2}\right\} c^{2}-2 c$, which is minimised when $d$ is chosen as $\theta_{0}$ and $c$ is chosen as $1 / \tau^{2}$. This provides fresh and independent motivation for using

$$
\theta_{n}^{*}=\frac{n}{n+1 / \tau^{2}} \bar{X}_{n}+\frac{1 / \tau^{2}}{n+1 / \tau^{2}} \theta_{0}
$$

in the presence of such prior knowledge, and agrees with both familiar Bayesian calculations in the normal model and the so-called credibility formula of actuarial statistics. Observe that $\theta_{n}^{*}$ can expect to make fewer $\varepsilon$-errors than $\bar{X}_{n}$ does if the true $\theta$ parameter is within $\sqrt{2} \tau$ of $\theta_{0}$. Note also that only $\theta_{0}$ and $\tau^{2}$ matter regarding the choice of prior weight function. Further discussion about risks and average risks is in Section 5 .

3B. Exponential mean. Let $X_{i} \sim \operatorname{Exp}(1 / \theta)$, and consider $Q_{\varepsilon}(c)$, the number of times $\left|\widehat{\theta}_{n}(c) / \theta-1\right| \geq \varepsilon$, where $\widehat{\theta}_{n}(c)=\frac{n}{n+c} \bar{X}_{n}$. This can be written $\left|\frac{n}{n+c} \bar{Y}_{n}-1\right| \geq \varepsilon$, where the $Y_{i}$ 's come from $e^{-y}$. This is as in Section 2 with $\xi=1, \sigma=1, \gamma=2$. The limit of interest is $c^{2}-\frac{2}{3} c$, with best value $c_{0}=\frac{1}{3}$. The ML solution with $c=0$ can be expected to make $\frac{1}{9}$ more $\varepsilon$-errors [sic] while the best estimator under squared error loss, which uses $c=1$, can be expected to make $\frac{4}{9}$ more $\varepsilon$-errors.

3C. Normal variance. Next consider $\widehat{\sigma}_{N}^{2}(c)=\sum_{i=1}^{N}\left(Y_{i}-\bar{Y}_{N}\right)^{2} /(N-1+c)$ for estimating the variance $\sigma^{2}$ based on data $Y_{1}, \ldots, Y_{N}$ that are normal $\left(\mu, \sigma^{2}\right)$. Study the number of times $\left|\widehat{\sigma}_{N}^{2}(c) / \sigma^{2}-1\right| \geq \varepsilon$. This can be written $\left|\frac{n}{n+c}\left(\chi_{n}^{2} / n\right)-1\right| \geq \varepsilon$, writing $n$ for $N-1$, the degrees of freedom. This is once more as in Section 2, this time with $\chi_{1}^{2}$ variables playing the rôle of $X_{i}$ 's. These have $\xi=1, \sigma=\sqrt{2}$ and $\gamma=2 \sqrt{2}$. The limit to study is $\frac{1}{2} c^{2}-\frac{2}{3} c$, with minimum occurring for $c_{0}=\frac{2}{3}$. Hence

$$
\widehat{\sigma}_{N}^{2}=\frac{1}{N-(1 / 3)} \sum_{i=1}^{N}\left(Y_{i}-\bar{Y}_{N}\right)^{2}
$$

makes the fewest $\varepsilon$-errors! See also (4.4) and (4.6) below.

3D. Binomial probability. Let $Y_{n}$ be binomial $(n, p)$, and let $Q_{\varepsilon}(c, d)$ count $\varepsilon$-misses for $\left(Y_{n}+c d\right) /(n+c)$. Note that $Y_{n}$ is the sum of $X_{i}$ 's with mean $p$, variance $p q$, and skewness $\gamma=(q-p) /(p q)^{1 / 2}$, where $q=1-p$. Hence the limit in (2.8) becomes

$$
\operatorname{risk}(c, d)=\frac{(p-d)^{2}}{p q} c^{2}-2 c-\frac{2}{3} \frac{(p-q)(p-d)}{p q} c .
$$

After studying the case with 'prior guess' $d=\frac{1}{2}$ one is lead to

$$
p_{n}^{*}=\frac{Y_{n}+2 / 3}{n+4 / 3}
$$


being the 'best' sequence. It can, seemingly, expect to make 2.667 fewer $\varepsilon$-errors than $Y_{n} / n$ does, regardless of $p$ !

There is an obstacle here, however, in that the distribution of $X_{i}$ is lattice, the Cramércondition does not hold, and fine-tuned corrections are necessary for a formula like (2.6) to hold. Techniques from Kolassa and McCullagh (1990) are likely to provide in the end a formula like (2.8) appropriate for the lattice case, but we have not pursued this. A possible trick to avoid these difficulties associated with exact a.r.d. calculations here is to spread the probability mass $p$ for $X_{i}$ uniformly on some tiny $[1-\eta, 1+\eta]$. Now results of Section 2 are in force, giving the best sequence of estimates for $p$. Letting $\eta \rightarrow 0$ in the end singles out the (3.3) sequence as the best. Note finally that the $\operatorname{Beta}(4 / 3,4 / 3)$ is a least favourable prior for $p$, in the sense of the calculations above.

4. Further examples. The aim of this section is to establish results in a couple of technically more demanding estimation problems. In each case somewhat strenuous modifications of the arguments used in Section 2 have to be devised in order to establish the limit of expected $Q_{\varepsilon}$-differences.

4A. The squared mean in the normal model: known variance. Let $X_{i}$ be normal $\left(\xi, \sigma^{2}\right)$, with $\sigma$ known, and suppose $\theta=\xi^{2}$ is to be estimated. The ML solution is $\left(\bar{X}_{n}\right)^{2}$, which could overestimate; the UMV solution is $\left(\bar{X}_{n}\right)^{2}-\sigma^{2} / n$. Study therefore $\widehat{\theta}_{n}(d)=$ $\left(\bar{X}_{n}\right)^{2}-d \sigma^{2} / n$ and its corresponding $Q_{\varepsilon}(d)$, the number of $\varepsilon$-misses among $n \geq a(\varepsilon) / \varepsilon^{2}$, with $a(\varepsilon)$ going to zero as in (2.7). We intend to show that

$$
\lambda_{0}(d)=\lim _{\varepsilon \rightarrow 0} E\left\{Q_{\varepsilon}(d)-Q_{\varepsilon}(0)\right\}=\left(\frac{1}{4} d^{2}+\frac{1}{2} d\right) \frac{\sigma^{2}}{\xi^{2}}
$$

Some alterations to the program of Section 2 are necessary. The indicator function summand in $Q_{\varepsilon}(d)$ is 1 if $\left|\left(\xi+\sigma_{n} / \sqrt{n}\right)^{2}-d \sigma^{2} / n-\xi^{2}\right| \geq 1 / \sqrt{m}$, in which $T_{n}=\sqrt{n}\left(\bar{X}_{n}-\xi\right) / \sigma$. This can be written

$$
T_{n}^{2}+2(\xi / \sigma) \sqrt{n} T_{n} \geq d+\sqrt{n} \sqrt{s} / \sigma^{2} \quad \text { or } \quad T_{n}^{2}+2\left(\xi / \sigma^{2}\right) \sqrt{n} T_{n} \leq d-\sqrt{n} \sqrt{s} / \sigma^{2}
$$

where $s=n / m$ again. Let $b(d)<r(d)$ be the two roots of the first inequality and $c(d)<l(d)$ the two roots of the second inequality. Assume now that $\xi$ is positive. Then $b(d)<c(d)<l(d)<0<r(d)$, and the indicator function term is

$$
I\left\{T_{n} \leq b(d) \text { or } T_{n} \geq r(d) \text { or } c(d) \leq T_{n} \leq l(d)\right\}
$$

It turns out that both $b(d)$ and $c(d)$ are of size about $-2(\xi / \sigma) \sqrt{n}$, which is too far out on the left to be of significance, and we can concentrate on $I\left\{T_{n} \leq l(d)\right.$ or $\left.T_{n} \geq r(d)\right\}$. We need to compute the limit of

$$
E\left\{Q_{\varepsilon}(d)-Q_{\varepsilon}(0)\right\} \doteq \sum_{n \geq a m}[\{\Phi(l(d))-\Phi(l(0))\}-\{\Phi(r(d))-\Phi(r(0))\}]
$$


cf. (2.4) and (2.5). A determined mind finds

$$
\begin{aligned}
l(d) & =-\sqrt{n} \frac{\xi}{\sigma}+\sqrt{n} \frac{\xi}{\sigma}\left(1-\frac{1}{\sqrt{n}} \frac{\sqrt{s}}{\xi^{2}}+\frac{1}{n} \frac{d \sigma^{2}}{\xi^{2}}\right)^{1 / 2} \\
& \doteq-\frac{1}{2} \frac{\sqrt{s}}{\xi \sigma}+\frac{1}{\sqrt{n}}\left(\frac{1}{2} \frac{d \sigma}{\xi}-\frac{1}{8} \frac{s}{\xi^{3} \sigma}\right)+\frac{1}{n}\left(\frac{1}{4} d \sqrt{s} \frac{\sigma}{\xi^{3}}-\frac{1}{16} \frac{s^{3 / 2}}{\xi^{5} \sigma}\right) \\
& =-u+\delta_{1}(d) / \sqrt{n}+\delta_{2}(d) / n \\
r(d) & =-\sqrt{n} \frac{\xi}{\sigma}+\sqrt{n} \frac{\xi}{\sigma}\left(1+\frac{1}{\sqrt{n}} \frac{\sqrt{s}}{\xi^{2}}+\frac{1}{n} \frac{d \sigma^{2}}{\xi^{2}}\right)^{1 / 2} \\
& \doteq \frac{1}{2} \frac{\sqrt{s}}{\xi \sigma}+\frac{1}{\sqrt{n}}\left(\frac{1}{2} \frac{d \sigma}{\xi}-\frac{1}{8} \frac{s}{\xi^{3} \sigma}\right)+\frac{1}{n}\left(-\frac{1}{4} d \sqrt{s} \frac{\sigma}{\xi^{3}}+\frac{1}{16} \frac{s^{3 / 2}}{\xi^{5} \sigma}\right) \\
& =u+\delta_{1}(d) / \sqrt{n}-\delta_{2}(d) / n .
\end{aligned}
$$

One can now compute Taylor approximations to the terms of (4.2). The result, apart from $O\left(n^{-3 / 2}\right)$-terms, is

$$
\begin{gathered}
2 \phi(u)\left\{\delta_{2}(d)-\delta_{2}(0)\right\} / n+\left\{\frac{1}{2} \phi^{\prime}(-u)-\frac{1}{2} \phi^{\prime}(u)\right\}\left\{\delta_{1}(d)^{2}-\delta_{1}(0)^{2}\right\} / n \\
=\frac{1}{m s} \phi\left(\frac{1}{2} \frac{\sqrt{s}}{\xi \sigma}\right)\left[\frac{1}{2} d \sqrt{s} \frac{\sigma}{\xi^{3}}+\left(\frac{1}{4} \frac{d^{2} \sigma^{2}}{\xi^{2}}-\frac{1}{8} \frac{d s}{\xi^{4}}\right) \frac{1}{2} \frac{\sqrt{s}}{\xi \sigma}\right] .
\end{gathered}
$$

Further care and attention to details lead to (4.1).

When $\xi<0$ then similar arguments give the same answer $\left(\frac{1}{4} d^{2}+\frac{1}{2} d\right) \sigma^{2} / \xi^{2}$. We may conclude that the choice $d_{0}=-1$ is best, that is, within the class of estimators under consideration, the estimator $\left(\bar{X}_{n}\right)^{2}+\sigma^{2} / n$ has the second order optimality property of making the fewest $\varepsilon$-errors, in expectation, in the limit as $\varepsilon \rightarrow 0$. The ML solution $\left(\bar{X}_{n}\right)^{2}$ can expect to make $\frac{1}{4} \sigma^{2} / \xi^{2}$ more $\varepsilon$-errors while the $\operatorname{UMV}\left(\bar{X}_{n}\right)^{2}-\sigma^{2} / n$ can expect to make $\sigma^{2} / \xi^{2}$ more $\varepsilon$-errors.

A natural extension is to include all estimators of the type $\widehat{\theta}_{n}(c, d)=\left(\frac{n}{n+c} \bar{X}_{n}\right)^{2}-$ $d \sigma^{2} / n$. Let $Q_{\varepsilon}(c, d)$ be its associated number of $\varepsilon$-misses. Then variations of the arguments above and the necessary stamina lead to

$$
\lambda_{0}(c, d)=\lim _{\varepsilon \rightarrow 0} E\left\{Q_{\varepsilon}(c, d)-Q_{\varepsilon}(0,0)\right\}=c^{2}\left(\xi^{2} / \sigma^{2}\right)+c d-3 c+\left(\frac{1}{4} d^{2}+\frac{1}{2} d\right)\left(\xi^{2} / \sigma^{2}\right) .
$$

This can be averaged with respect to a weight function for $\xi$-values, to give

$$
A c^{2}+c d-3 c+B\left(\frac{1}{4} d^{2}+\frac{1}{2} d\right), \quad \text { where } A=E\left(\xi^{2} / \sigma^{2}\right) \text { and } B=E\left(\sigma^{2} / \xi^{2}\right)
$$

see Section 5 for this type of average risk reasoning. Minimising this singles out

$$
c_{0}=\frac{2 B}{A B-1} \quad \text { and } \quad d_{0}=-\frac{A B+3}{A B-1}
$$

Note that $c_{0}>0$ while $d_{0}<0$. 
REMARK. The asymptotics work differently in the special case $\xi=0$. In this case $\sqrt{n}\left\{\widehat{\theta}_{n}(c, d)-\theta\right\}$ goes to zero, as does $\varepsilon^{2} Q_{\varepsilon}(c, d)$. By methods of Hjort and Fenstad (1991) one can prove that

$$
\varepsilon Q_{\varepsilon}(c, d) \rightarrow_{d} Q^{*}=\int_{0}^{\infty} I\left\{\sigma^{2}\left|W(s)^{2} / s^{2}-d / s\right| \geq 1\right\} \mathrm{d} s
$$

a variable with mean value $\sigma^{2} E\left|N(0,1)^{2}-d\right|$. So in this null case $d=-1$ is not as good as $d=0$ and $d=1$. One can work out that this limit mean is smallest for $\sqrt{d_{0}}=\Phi^{-1}(0.75)$, i.e. when $d_{0}=0.455$.

4B. The squared mean in the normal model: unknown variance. The extension to unknown $\sigma$ is important because this parameter usually is unknown, of course, but also because the techniques given in a moment serve to illustrate how similar problems can be solved in other two- and multi-parameter situations.

The natural class of estimators to study is $\theta_{n}^{*}(d)=\left(\bar{X}_{n}\right)^{2}-d \widehat{\sigma}_{n}^{2} / n$, in which we take $\widehat{\sigma}_{n}^{2}=\sum_{i=1}^{n}\left(X_{i}-\bar{X}_{n}\right)^{2} /(n-1)$ to be the unbiased version. Again $d=0$ gives the ML solution while $d=1$ gives the UMV estimator. Write $\widehat{\sigma}_{n}^{2}=\sigma^{2} Z_{n}$, where $Z_{n}$ is independent of $\bar{X}_{n}$ and distributed as $\chi_{n-1}^{2} /(n-1)$, so that in fact $\theta_{n}^{*}(d)=\widehat{\theta}_{n}\left(d Z_{n}\right)$, in the notation of $4 \mathrm{~A}$. The arguments there show that $Q_{\varepsilon}^{*}(d)$, the number of cases where $\left|\theta_{n}^{*}(d)-\xi^{2}\right| \geq \varepsilon$, essentially counts cases where $T_{n} \leq l\left(d Z_{n}\right)$ or $T_{n} \geq r\left(d Z_{n}\right)$. The analogue of (4.2) becomes

$$
\sum_{n \geq a m}\left\{\left[\operatorname{Pr}\left\{T_{n} \leq l\left(d Z_{n}\right)\right\}-\operatorname{Pr}\left\{T_{n} \leq l(0)\right\}\right]-\left[\operatorname{Pr}\left\{T_{n} \leq r\left(d Z_{n}\right)\right\}-\operatorname{Pr}\left\{T_{n} \leq r(0)\right\}\right]\right\} .
$$

The simplest way to compute the limit of this sum, as $m=1 / \varepsilon^{2}$ grows, is to condition on the value of $Z_{n}$, use results of $4 \mathrm{~A}$, and then integrate over the distribution $g_{n}\left(z_{n}\right)$ for $Z_{n}$. Carrying through this gives

$$
\sum_{n \geq a m} \int_{0}^{\infty} \frac{1}{m s} \phi\left(\frac{1}{2} \frac{\sqrt{s}}{\xi \sigma}\right)\left[\frac{1}{2} d \sqrt{s} z_{n} \frac{\sigma}{\xi^{3}}+\frac{1}{2} \frac{\sqrt{s}}{\xi \sigma}\left(\frac{1}{4} d^{2} z_{n}^{2} \frac{\sigma^{2}}{\xi^{2}}-\frac{1}{8} d z_{n} \frac{s}{\xi^{4}}\right)\right] g_{n}\left(z_{n}\right) \mathrm{d} z_{n} .
$$

But since $E Z_{n}=1$ and $E Z_{n}^{2}=1+2 /(n-1)$ the limit becomes in the end equal to the previous answer $\left(\frac{1}{4} d^{2}+\frac{1}{2} d\right) \sigma^{2} / \xi^{2}$. We may conclude that $\left(\bar{X}_{n}\right)^{2}+\widehat{\sigma}_{n}^{2} / n$ is best.

It is interesting to compare this result to the corresponding one using the Hodges and Lehmann deficiency. Calculations with the mean squared error of $\theta_{n}^{*}(d)$ show that a.r.d.hl, the limit of the sample size difference $n_{0}(d)-n_{0}(0)$, becomes $\left(\frac{1}{4} d^{2}-\frac{1}{2} d\right) \sigma^{2} / \xi^{2}$, and the best estimator with this criterion is the UMV solution $\left(\bar{X}_{n}\right)^{2}-\widehat{\sigma}_{n}^{2} / n$.

4C. Standard deviation in the normal model. Let us next present a variant of Example $3 C$. Instead of counting instances of $\left|\widehat{\sigma}_{N}^{2}(c) / \sigma^{2}-1\right| \geq \varepsilon$, penalise errors using the natural scale for $\sigma$ and study

$$
Q_{\varepsilon}(c)=\sum_{N \geq a / \varepsilon^{2}} I\left\{\left|\widehat{\sigma}_{N}(c) / \sigma-1\right| \geq \varepsilon\right\}
$$

The finer aspects of the second order asymptotics machinery turn out to give a different answer than in $3 \mathrm{C}$ for the best value of $c$. To find the best value this time, write $Q_{\varepsilon}(c)$ as 
the number of times $\left|\left(\frac{n}{n+c} \bar{X}_{n}\right)^{1 / 2}-1\right| \geq \varepsilon$, where $n=N-1$ again and $\bar{X}_{n}$ is the average of nonnegative variables $X_{i}$ with mean $\xi=1$, standard deviation $\tau=\sqrt{2}$, and skewness $\gamma=2 \sqrt{2}$. Using $T_{n}=\sqrt{n}\left(\bar{X}_{n}-1\right) / \tau$ again one can show that $\left|\left\{\frac{n}{n+c}\left(1+\tau T_{n} / \sqrt{n}\right)\right\}^{1 / 2}-1\right| \geq$ $1 / \sqrt{m}$ becomes equivalent to $T_{n} \geq r(c)$ or $T_{n} \leq l(c)$, in which

$$
r(c)=u+\frac{\delta_{1}(c)}{\sqrt{m}}+\frac{\delta_{2}(c)}{m} \text { and } l(c)=-u+\frac{\delta_{1}(c)}{\sqrt{m}}-\frac{\delta_{2}(c)}{m}
$$

where

$$
u=2 \sqrt{s} / \tau, \quad \delta_{1}(c)=(c / \sqrt{s}+\sqrt{s}) / \tau, \quad \delta_{2}(c)=(2 c / \sqrt{s}) / \tau
$$

and $s=n / m$ once more. A variant of the Taylor expansion that followed (2.5) can be established for the present occasion, and reads

$$
G_{n}\left(t+\varepsilon_{2}\right)-G_{n}\left(t+\varepsilon_{1}\right)=\phi(t)\left(\varepsilon_{2}-\varepsilon_{1}\right)+\frac{1}{2} \phi^{\prime}(t)\left(\varepsilon_{2}^{2}-\varepsilon_{1}^{2}\right)+\frac{1}{6} \frac{\gamma}{\sqrt{n}} B(t) \phi(t)\left(\varepsilon_{2}-\varepsilon_{1}\right)+O\left(n^{-3 / 2}\right)
$$

This formula is valid if $\varepsilon_{1}$ and $\varepsilon_{2}$ are of order $1 / \sqrt{n}$, and leads to expressions for $G_{n}(l(c))-$ $G_{n}(l(0))$ and $G_{n}(r(c))-G_{n}(r(0))$, in terms of $\delta_{1}(0), \delta_{1}(c), \delta_{2}(0), \delta_{2}(c)$. The result is that the terms of the current analogue to $(2.5)$ can be written

$$
\frac{1}{m} \phi(u)\left[-2 \frac{2 c}{\sqrt{s}} \frac{1}{\tau}+u \frac{1}{\tau^{2}}\left(\frac{c^{2}}{s}+2 c\right)-\frac{1}{3} \frac{\gamma}{\sqrt{s}} B(u) \frac{1}{\tau} \frac{c}{\sqrt{s}}\right]+O\left(n^{-3 / 2}\right)
$$

where $u=2 \sqrt{s} / \tau$ is to be inserted. The infinite sum over $n / m \geq a$ becomes an integral over $s \geq a$ in the limit as $m=1 / \varepsilon^{2}$ goes to inifinity. The limit is

$$
\int_{2 \sqrt{a} / \tau}^{\infty} \phi(u)\left[-8 c+u^{2}\left(\frac{4 c^{2}}{\tau^{2} u^{2}}+2 c\right)-\frac{4}{3} \gamma \frac{c}{\tau} \frac{B(u)}{u}\right] \frac{1}{2} \mathrm{~d} u
$$

In the $a(\varepsilon)=\varepsilon$ case the integration is over the full halfline, and becomes

$$
\lambda_{0}(c)=\lim _{\varepsilon \rightarrow 0} E\left\{Q_{\varepsilon}(c)-Q_{\varepsilon}(0)\right\}=\frac{c^{2}}{\tau^{2}}+\left(\frac{2}{3} \frac{\gamma}{\tau}-\frac{3}{2}\right) c
$$

In the particular $X_{i} \sim \chi_{1}^{2}$ case under study we find $\lambda_{0}(c)=\frac{1}{2} c^{2}-\frac{1}{6} c$, with minimum occurring for $c_{0}=\frac{1}{6}$. We may conclude that the estimator sequence

$$
\tilde{\sigma}_{N}=\left\{\frac{1}{N-(5 / 6)} \sum_{i=1}^{N}\left(Y_{i}-\bar{Y}_{N}\right)^{2}\right\}^{1 / 2}
$$

can be expected to make fewest errors of the type $\left|\widehat{\sigma}_{N} / \sigma-1\right| \geq \varepsilon$.

The arguments above can be used to establish a modest but useful generalisation of (2.7) and (2.8). Suppose $h(\xi)$ is a smooth increasing transformation, and consider $Q_{\varepsilon}^{*}(c, d)$, the number of times $\left|h\left(\widehat{\xi}_{n}(c, d)\right)-h(\xi)\right| \geq \varepsilon$, among $n \geq 1 / \varepsilon$ (see (2.7)), where 
$\widehat{\xi}_{n}(c, d)=\left(n \bar{X}_{n}+c d\right) /(n+c)$ as in Proposition 3 of Section 2. One can go through previous arguments, supplement them with further Taylor analysis, and prove

$$
\lambda_{0}^{*}(c, d)=\lim _{\varepsilon \rightarrow 0} E\left\{Q_{\varepsilon}^{*}(c, d)-Q_{\varepsilon}^{*}(0,0)\right\}=\frac{(\xi-d)^{2}}{\sigma^{2}} c^{2}+\left\{-2+\frac{2 \gamma}{3} \frac{\xi-d}{\sigma}+\frac{k_{2}}{k_{1}^{2}}(\xi-d)\right\} c,
$$

in which $k_{1}=\left(h^{-1}\right)^{\prime}(h(\xi))$ and $k_{2}=\left(h^{-1}\right)^{\prime \prime}(h(\xi))$. Elementary rules show that $k_{2} / k_{1}^{2}$ also can be written as $-h^{\prime \prime}(\xi) / h^{\prime}(\xi)$. The special case $h(\xi)=\xi$ has $k_{2}=0$ and gives back (2.8).

The $\lambda_{0}(c)$ result above can be shown from this, using $h(\xi)=\xi^{1 / 2}, d=0$, and using $\xi=1$ in the end. Let us also use (4.5) to exhibit the estimator sequence that can be expected to produce the fewest $\varepsilon$-errors on $\log$ scale. So let $Q_{\varepsilon}^{*}(c)$ count instances of $\left|\log \widehat{\sigma}_{N}^{2}(c)-\log \sigma^{2}\right| \geq \varepsilon$. Then the limit can be shown to be $\lambda_{0}^{*}(c)=\frac{1}{2} c^{2}-\left(\frac{2}{3}-e^{-1}\right) c$, with minimum for $c_{0}=\frac{2}{3}-e^{-1}$. So using denominator $N-1+c_{0}$, i.e.

$$
\sigma_{N}^{*}=\left\{\frac{1}{N-0.695} \sum_{i=1}^{N}\left(Y_{i}-\bar{Y}_{N}\right)^{2}\right\}^{1 / 2}
$$

can be expected to make fewest errors for $\sigma^{p}$ on the log scale, for every value of $p$.

REmarK. Formulae (3.2), (4.4) and (4.6) add three new items to the distinguished list of denominators in the variance estimation formula $\sum_{i=1}^{N}\left(Y_{i}-\bar{Y}_{n}\right)^{2} /(N-1+c)$ reached by various statistical principles. This list includes: (i) The maximum likelihood estimator, normal-based or nonparametric, has $1 / N$. (ii) The unbiased estimator for $\sigma^{2}$ with smallest variance, under normality or under nonparametric circumstances, has $1 /(N-1)$. (iii) If one wants $E \widehat{\sigma}_{N}(c)=\sigma$, unbiasedness on natural scale, then one needs $E\left(\chi_{n}^{2}\right)^{1 / 2} /(n+c)^{1 / 2}=1$, using $n=N-1$ again, whose approximate solution is $c=-\frac{1}{2}$, leading to $1 /\left(N-\frac{3}{2}\right)$. (iv) Another possibility is to minimise mean squared error $E_{\sigma}\left(\widehat{\sigma}_{N}^{2}-\sigma^{2}\right)^{2}$. The best constant is $1 /(N+1)$. This also gives the best invariant estimator under squared error loss for $\sigma^{2}$. (v) It appears as natural to solve this problem using natural scale, i.e. to mimimise $E_{\sigma}\left(\widehat{\sigma}_{N}-\sigma\right)^{2}$. This can again be done uniformly in $\sigma$, and the solution is $c=n^{2} /\left\{E\left(\chi_{n}^{2}\right)^{1 / 2}\right\}^{2}-n$, for which $\frac{1}{2}$ is an approximation. The best denominator is $N-\frac{1}{2}$, and gives at the same time the best invariant estimator under squared error loss for $\sigma$. (vi) The median of $\chi_{n}^{2}$ is $n-2 / 3+(4 / 27) / n$ to a good approximation, by the Wilson-Hilferty formula. Hence denominator $N-\frac{5}{3}+\frac{4}{27 N} \doteq N-\frac{5}{3}$ gives an approximate median-unbiased estimator; it overshoots as often as it undershoots. (vii) Since $E \log \left(\chi_{n}^{2}\right)=\log 2+\psi(n / 2) \doteq \log n-$ $\left(\frac{1}{n}+\frac{1}{3 n^{2}}\right)$ one finds that $\widetilde{\sigma}$ with denominator $N-2+\frac{1}{6 N} \doteq N-2$ gives unbiasedness on $\log$ scale. This is a good property both because $\log \sigma$ is a good quantity and because $\tilde{\sigma}^{p}$ becomes $\log$-unbiased for $\sigma^{p}$ for every value of $p$. (viii) The Bayes solution under a vague prior ( $\log \sigma$ uniform on the line) uses $N-3$ if loss is squared error on $\sigma^{2}$ scale and $N-1$ if loss is squared error on $1 / \sigma^{2}$ scale.

\section{Loss functions and Bayes solutions.}

5A. Decision theoretic framework. Who can understand his errors (Psalm $19: 12)$ ? And who can count them? We are essentially working with the somewhat non-standard loss function $L_{\varepsilon}$ that for a given sequence of estimates $\left\{\widehat{\theta}_{n}\right\}$ counts

$$
L_{\varepsilon}\left[\theta,\left\{\hat{\theta}_{n}: n \geq 1\right\}\right]=\sum_{n \geq a(\varepsilon) / \varepsilon^{2}} I\left\{\left|\widehat{\theta}_{n}-\theta\right| \geq \varepsilon\right\}
$$


the number of $\varepsilon$-errors among all $n \geq a(\varepsilon) / \varepsilon^{2}$ cases, for a very small $\varepsilon$. The associated risk function is

$$
R_{\varepsilon}(\theta)=E_{\theta} L_{\varepsilon}\left[\theta,\left\{\widehat{\theta}_{n}: n \geq 1\right\}\right]=\sum_{n \geq a(\varepsilon) / \varepsilon^{2}} \operatorname{Pr}_{\theta}\left\{\left|\widehat{\theta}_{n}-\theta\right| \geq \varepsilon\right\}
$$

In some cases this series diverges while the difference risk $R_{1, \varepsilon}(\theta)-R_{2, \varepsilon}(\theta)$ (say) might constitute a convergent series. The $\lambda_{a}(c)$ and $\lambda_{0}(c)$ functions found in Section 2 are indeed limits of $R_{c, \varepsilon}(\theta)-R_{0, \varepsilon}(\theta)$ as $\varepsilon \rightarrow 0$, where $R_{c, \varepsilon}$ refers to the risk function for the $\widehat{\theta}_{n}(c)$ sequence. One might next obtain average risk with respect to a weight function over the parameter space, which one may choose or not choose to interpret as a prior distribution in the Bayesian fashion, and finally minimise this expression w.r.t. the class of estimators under consideration.

This was the program partially carried out in Examples $3 \mathrm{~A}$ and $4 \mathrm{~A}$. A more direct and general approach which avoids restriction to a given class of estimators is possible, as follows, provided the prior is fully specified. Let $\pi($.$) be the prior distribution and$ $\pi_{n}()=.\pi_{n}\left(. \mid x_{1}, \ldots, x_{n}\right)$ the posterior distribution at step $n$. Then

$$
\int R_{\varepsilon}(\theta) \pi(\theta) \mathrm{d} \theta=\sum_{n \geq a / \varepsilon^{2}} \int \cdots \int \pi_{n}\left\{\left|\theta-\widehat{\theta}_{n}\right| \geq \varepsilon\right\} f_{n}\left(x_{1}, \ldots, x_{n}\right) \mathrm{d} x_{1} \cdots \mathrm{d} x_{n}
$$

where $f_{n}$ is the marginal density resulting from having $\pi($.$) as prior. For given \varepsilon$ the best solution is to minimise each term, which means choosing $\widehat{\theta}_{n}$ to maximise $\pi_{n}\left\{\left|\theta-\widehat{\theta}_{n}\right| \leq \varepsilon\right\}$, for each given $x_{1}, \ldots, x_{n}$. When $\varepsilon$ goes to zero for fixed $n$ this would mean using the posterior mode. The balance is more delicate here, however, where summing over $n$ comes before letting $\varepsilon$ tend to zero. Think of $\widehat{\theta}_{n}$ as $\theta_{n}^{*}+u / \sqrt{n}$, where $\theta_{n}^{*}$ is the posterior mean for $\theta$. Thus one needs to minimise $\pi_{n}\left\{\left|\sqrt{n}\left(\theta-\theta_{n}^{*}\right)-u\right| \geq \sqrt{s}\right\}$ w.r.t. $u$, where $m=1 / \varepsilon^{2}$ and $s=n / m$ as in earlier sections. A full analysis would call for Edgeworth expansions again. Observe in particular that the skewness of the posterior distribution plays a rôle here.

5B. A normal mean. Consider once more the situation of $3 \mathrm{~A}$, where it was noted that $\theta_{n}^{*}$ of (3.1) was best among all linear functions of the sample mean, for any prior weight function with first moment $\theta_{0}$ and variance $\tau^{2}$. Suppose now that $\theta$ is given the normal prior with these parameters. Then $\pi_{n}($.$) is also normal, with mean equal to \theta_{n}^{*}$ of (3.1) and variance $\tau_{n}^{2}=\tau^{2} /\left(n \tau^{2}+1\right)$. The symmetry of the normal density shows that $\pi_{n}\left\{\left|\theta-\widehat{\theta}_{n}\right| \leq \varepsilon\right\}$ is maximised for $\widehat{\theta}_{n}=\theta_{n}^{*}$. So (3.1) is not only the best solution within the linear class $\widehat{\theta}_{n}(c, d)$ but the very best of all estimators, under the average $E Q_{\varepsilon}$ criterion, provided the prior used is indeed $N\left\{\theta_{0}, \tau^{2}\right\}$. This even holds for each positive $\varepsilon$ as well as in the limit.

6. A fuller story: Second order limits in distribution. Let us go back to the situation of Section 2. Take $c$ and $\xi$ to be positive so that $l(c)>l(0)$ and $r(c)>r(0)$ in (2.3). It follows from (2.4) that

$$
Q_{\varepsilon}(c)-Q_{\varepsilon}(0)=\sum_{n \geq a m}\left[I\left\{l(0) \leq T_{n} \leq l(c)\right\}-I\left\{r(0) \leq T_{n} \leq r(c)\right\}\right]
$$


This is an infinite sum of quite rare 1's and -1 's. We have so far only been working with the expected value of this difference, and stayed within the realm of probabilities and Edgeworth expansions. There is also a fuller story to be told, involving second order distributional aspects of the two sums in (6.1).

To investigate these aspects, introduce

$$
W_{m}(s)=\frac{1}{\sqrt{m}} \sum_{i=1}^{[m s]}\left(X_{i}-\xi\right) / \sigma=\left(\frac{[m s]}{m}\right)^{1 / 2} T_{[m s]}
$$

which converges to Brownian motion $W(s)$ by Donsker's theorem. Write the random $Q_{\varepsilon}(c)-Q_{\varepsilon}(0)$ as $\sqrt{m}\left(A_{m}-B_{m}\right)$, where $m=1 / \varepsilon^{2}$ again, and

$$
\begin{aligned}
A_{m} & =\frac{1}{\sqrt{m}} \sum_{n \geq a m} I\left\{-\sqrt{\frac{n}{m}} \frac{1}{\sigma} \leq \sqrt{n}\left(\bar{X}_{n}-\xi\right) / \sigma \leq-\sqrt{\frac{n}{m}} \frac{1}{\sigma}+\frac{1}{\sqrt{n}} \frac{c \xi}{\sigma}-\frac{1}{\sqrt{n m}} \frac{c}{\sigma}\right\} \\
& =\frac{1}{\sqrt{m}} \sum_{n \geq a m} I\left\{-\frac{n}{m} \frac{1}{\sigma} \leq W_{m}(s) \leq-\frac{n}{m} \frac{1}{\sigma}+\frac{1}{\sqrt{m}} \frac{c \xi}{\sigma}-\frac{1}{m} \frac{c}{\sigma}\right\} \\
& =\sqrt{m} \int_{\langle a m\rangle / m}^{\infty} I\left\{-\frac{[m s]}{m} \frac{1}{\sigma} \leq W_{m}(s) \leq-\frac{[m s]}{m} \frac{1}{\sigma}+\frac{1}{\sqrt{m}} \frac{c \xi}{\sigma}-\frac{1}{m} \frac{c}{\sigma}\right\} \mathrm{d} s,
\end{aligned}
$$

and similarly

$$
\begin{aligned}
B_{m} & =\frac{1}{\sqrt{m}} \sum_{n \geq a m} I\left\{\sqrt{\frac{n}{m}} \frac{1}{\sigma} \leq \sqrt{n}\left(\bar{X}_{n}-\xi\right) / \sigma \leq \sqrt{\frac{n}{m}} \frac{1}{\sigma}+\frac{1}{\sqrt{n}} \frac{c \xi}{\sigma}+\frac{1}{\sqrt{n m}} \frac{c}{\sigma}\right\} \\
& =\sqrt{m} \int_{\langle a m\rangle / m}^{\infty} I\left\{\frac{[m s]}{m} \frac{1}{\sigma} \leq W_{m}(s) \leq \frac{[m s]}{m} \frac{1}{\sigma}+\frac{1}{\sqrt{m}} \frac{c \xi}{\sigma}+\frac{1}{m} \frac{c}{\sigma}\right\} \mathrm{d} s .
\end{aligned}
$$

Here $\langle a m\rangle$ denotes the smallest integer exceeding or equal to am. Write

$$
A_{m}=A_{m}^{*}-A_{m}^{0} \quad \text { and } \quad B_{m}=B_{m}^{*}+B_{m}^{0}
$$

where $A_{m}^{*}$ and $B_{m}^{*}$ are as in the definitions of $A_{m}$ and $B_{m}$ above but without the final $1 / m$ term, and $A_{m}^{0}$ and $B_{m}^{0}$ are the necessary corrections; see the expression for $B_{m}^{*}$ appearing in the proof below.

Our first point is that these integral representations lead to another way of proving (2.1) and (2.7), the main results of Section 1. The idea is to use (2.6) to obtain an approximation to $f_{m, s}( \pm s / \sigma)$ and its derivative, where $f_{m, s}(x)$ is the density of $W_{m}(s)$, after which patient Taylor type analysis gives

$$
\begin{aligned}
& E A_{m}^{*}=c \xi \alpha_{m}+m^{-1 / 2}\left\{\frac{1}{2}\left(c^{2} \xi^{2} / \sigma^{2}\right) \alpha_{m}-\frac{1}{6} \gamma(c \xi / \sigma) \beta_{m}\right\}+O\left((m \sqrt{a})^{-1}\right), \\
& E B_{m}^{*}=c \xi \alpha_{m}+m^{-1 / 2}\left\{-\frac{1}{2}\left(c^{2} \xi^{2} / \sigma^{2}\right) \alpha_{m}+\frac{1}{6} \gamma(c \xi / \sigma) \beta_{m}\right\}+O\left((m \sqrt{a})^{-1}\right), \\
& E A_{m}^{0}=m^{-1 / 2} c \alpha_{m}+O\left((m \sqrt{a})^{-1}\right), \\
& E B_{m}^{0}=m^{-1 / 2} c \alpha_{m}+O\left((m \sqrt{a})^{-1}\right),
\end{aligned}
$$


in which

$$
\begin{aligned}
\alpha_{m} & =\frac{1}{m} \sum_{n \geq a m} \frac{1}{\sigma} \phi\left(\sqrt{\frac{n}{m}} \frac{1}{\sigma}\right)\left(\frac{n}{m}\right)^{-1 / 2} \\
& \rightarrow \int_{a}^{\infty} \frac{1}{\sigma} \phi\left(\frac{\sqrt{s}}{\sigma}\right) \frac{\mathrm{d} s}{\sqrt{s}}=\int_{\sqrt{a} / \sigma}^{\infty} 2 \phi(x) \mathrm{d} x=\alpha(a), \\
\beta_{m} & =\frac{1}{m} \sum_{n \geq a m} \phi\left(\sqrt{\frac{n}{m}} \frac{1}{\sigma}\right) B\left(\sqrt{\frac{n}{m}} \frac{1}{\sigma}\right) / \frac{n}{m} \\
& \rightarrow \int_{a}^{\infty} \phi\left(\frac{\sqrt{s}}{\sigma}\right) B\left(\frac{\sqrt{s}}{\sigma}\right) \frac{\mathrm{d} s}{s}=\int_{\sqrt{a} / \sigma}^{\infty} 2 \phi(x) B(x) / x \mathrm{~d} x=\beta(a) .
\end{aligned}
$$

It follows that

$$
\begin{aligned}
E\left(A_{m}-B_{m}\right) & =E\left(A_{m}^{*}-B_{m}^{*}-A_{m}^{0}-B_{m}^{0}\right) \\
& =\frac{1}{\sqrt{m}}\left[\frac{c^{2} \xi^{2}}{\sigma^{2}} \alpha_{m}-\frac{1}{3} \gamma \frac{c \xi}{\sigma} \beta_{m}-2 c \alpha_{m}\right]+O\left((m \sqrt{a})^{-1}\right) .
\end{aligned}
$$

This proves (2.1) and (2.7) again, since $\alpha_{m}=\alpha(a)+O(1 / m)$ and $\beta_{m}=\beta(a)+O(1 / m)$, and the slightly more general $(2.8)$ can be proved similarly.

Our second and more important point is that limit distributions for $A_{m}$ and $B_{m}$ can be obtained. For a Brownian motion process $W(s)$, consider the variable

$$
V_{\delta}(a, b)=\frac{1}{\delta} \int_{a}^{\infty} I\{b s \leq W(s) \leq b s+\delta\} \mathrm{d} s
$$

Hjort (1991) shows that there is a well-defined limit variable $V(a, b)$ as $\delta \rightarrow 0$, which may be regarded as the total relative time spent by the Brownian motion process along the ray $w=b s$, during $s \in[a, \infty)$. He also shows that

$$
V(a, b) \sim k(|b| \sqrt{a}) \operatorname{Exp}(|b|)+(1-k(|b| \sqrt{a})) I_{0},
$$

in which $k(z)=\int_{z}^{\infty} 2 \phi(x) \mathrm{d} x$ and $I_{0}$ denotes unit point mass at zero. If in particular $a=0$, then $k(|b| \sqrt{a})=1$, and $V(0, b)$, the total relative time spent along $w=b s$, is simply exponentially distributed with parameter $|b|$.

Certain probabilities $\pi_{i j}$ appear in the theorem below. They are related to results of Hjort (1991, Sections 4 and 5), and can be found from

$$
\pi_{00}=H(\sqrt{a} / \sigma), \quad \pi_{01}=\pi_{10}=2 \Phi(\sqrt{a} / \sigma)-1-\pi_{00}, \quad \pi_{11}=1-\pi_{00}-\pi_{01}-\pi_{10},
$$

in which $H(z)$ is the probability that $\max _{0 \leq s \leq 1}|W(s)| \leq z$.

THEOREM. (i) If $a$ is fixed and positive, then

$$
\begin{gathered}
\left(A_{m}, B_{m}\right) \rightarrow_{d}(A, B)=((c \xi / \sigma) V(a,-1 / \sigma),(c \xi / \sigma) V(a, 1 / \sigma)), \\
\varepsilon\left\{Q_{\varepsilon}(c)-Q_{\varepsilon}(0)\right\}=A_{m}-B_{m}=\lambda_{a}(c) / \sqrt{m}+D_{m}+O_{p}(1 / m) \rightarrow_{d} A-B,
\end{gathered}
$$


as $\varepsilon=1 / \sqrt{m} \rightarrow 0$. Here $\lambda_{a}(c)$ is as in (2.1), and $D_{m}$ is a variable having mean zero and tending to $A-B$ in distribution. Furthermore

$$
A-B= \begin{cases}0 & \text { with probability } \pi_{00}, \\ c \xi U_{1} & \text { with probability } \pi_{10}, \\ -c \xi U_{2} & \text { with probability } \pi_{01}, \\ c \xi\left(U_{1}-U_{2}\right) & \text { with probability } \pi_{11}\end{cases}
$$

where $U_{1}$ and $U_{2}$ are dependent exponential variables with parameter 1. (ii) If $a=a(\varepsilon)=\varepsilon$, then

$$
\left(A_{m}, B_{m}\right) \text { tends to }(A, B)=((c \xi / \sigma) V(0,-1 / \sigma),(c \xi / \sigma) V(0,1 / \sigma))=\left(c \xi U_{1}, c \xi U_{2}\right)
$$

in distribution, where $U_{1}$ and $U_{2}$ are unit exponentials with intercorrelation $-\frac{1}{3}$.

PROOF: With $\delta=(c \xi / \sigma) / \sqrt{m}$ we may write

$$
B_{m}^{*}=\frac{c \xi}{\sigma} \frac{1}{\delta} \int_{\langle a m\rangle / m}^{\infty} I\left\{\frac{[m s]}{m} \frac{1}{\sigma} \leq W_{m}(s) \leq \frac{[m s]}{m} \frac{1}{\sigma}+\delta\right\} \mathrm{d} s .
$$

Hence $B_{m}^{*}$ is $c \xi / \sigma$ times a quantity which should be close in distribution to $V_{\delta}(a, 1 / \sigma)$ of (6.5), and therefore close to $V(a, 1 / \sigma)$ of (6.6). That indeed $\left(A_{m}^{*}, B_{m}^{*}\right)$ converges in distribution to the indicated $(A, B)$ limit follows from results in Hjort (1991, Section 5$)$, also in the more complicated $a(\varepsilon)=\varepsilon \rightarrow 0$ case. The methods there can also be used to demonstrate that $\sqrt{m} A_{m}^{0}$ and $\sqrt{m} B_{m}^{0}$ have limit distributions, i.e. $A_{m}^{0}$ and $B_{m}^{0}$ are both $O_{p}(1 / \sqrt{m})$, cf. (6.3). Representation (6.7) follows from this, and $\left(A_{m}, B_{m}\right)$ goes to $(A, B)$ too. Correlation and other simultaneous aspects of $(A, B)$ are discussed in Hjort (1991).

Note that $k(\sqrt{a} / \sigma)$ is equal to $\alpha(a)$, used in (6.3) and (6.4). Note also that the scaling property for Brownian motion $[W(c s) / \sqrt{c}$ defines a new Brownian motion, for each positive $c$ implies

$$
(A, B) \doteq_{d}\left(c \xi V\left(a / \sigma^{2},-1\right), c \xi V\left(a / \sigma^{2}, 1\right)\right)
$$

and finally that

$$
\begin{aligned}
& A_{m} \rightarrow_{d} A \sim \alpha(a) c \xi U_{1}+(1-\alpha(a)) I_{0}, \\
& B_{m} \rightarrow_{d} B \sim \alpha(a) c \xi U_{2}+(1-\alpha(a)) I_{0},
\end{aligned}
$$

where $U_{1}$ and $U_{2}$ are dependent and exponentially distributed with parameter 1.

The theorem gives a more complete picture of what happens to $Q_{\varepsilon}(c)-Q_{\varepsilon}(0)$ when $\varepsilon \rightarrow 0$. The first order result is that $\varepsilon^{2}\left\{Q_{\varepsilon}(c)-Q_{\varepsilon}(0)\right\}$ tends to zero in probability. On the second order level $\varepsilon\left\{Q_{\varepsilon}(c)-Q_{\varepsilon}(0)\right\}$ goes to $A-B$, which is informative, but doesn't really distinguish between the two competing estimator sequences, since $A-B$ and $B-A$ have the same distribution. On the third order level, however, $Q_{\varepsilon}(c)-Q_{\varepsilon}(0)$ has mean value going to $\lambda_{a}(c)$, but its distribution explodes, by (6.7). Consider the $\sigma^{2}$-estimation problem of $3 \mathrm{C}$, for example, with $a(\varepsilon)=\varepsilon \rightarrow 0$. Then the difference has mean value going to $\frac{1}{2} c^{2}-\frac{2}{3} c$, but its distribution is close to that of $\left(U_{1}-U_{2}\right) / \varepsilon$, which goes to $+\infty$ or $-\infty$ with equal probabilities $\frac{1}{2}$ and $\frac{1}{2}$. 


\section{References}

Barndorff-Nielsen, O.E. and Cox, D.R. (1989). Asymptotic Techniques for Use in Statistics. Chapman and Hall, London.

Berkson, J. (1980). Minimum chi-square, not maximum likelihood! (with discussion). Ann. Statist. 8, 457-487.

Ghosh, J.K. and Subramanyam, K. (1974). Second order efficiency of maximum likelihood estimators. Sankhyā, Ser. A 36, 325-258.

Hodges, J. and Lehmann, E.L. (1970). Deficiency. Ann. Math. Statist. 41, 783-801.

Hjort, N.L. and Fenstad, G. (1991). On the last time and the number of times an estimator is more than $\varepsilon$ from its target value. To appear in Ann. Statist.

Hjort, N.L. (1991). Some exponential distributions associated with Brownian motion. Statistical Research Report, University of Oslo; submitted for publication.

Kolassa, J.E. and McCullagh, P. (1990). Edgeworth series for lattice distributions. Ann. Statist. 18, 981-985.

Lehmann, E.L. (1983). Theory of Point Estimation. Wiley, New York.

Pfanzagl, J. (1973). Asymptotic expansions related to minimum contrast estimators. Ann. Statist. 1, 993-1026.

Rao, C.R. (1962). Efficient estimates and optimum inference procedures in large samples (with discussion). J. Roy. Statist. Soc., Ser. B 24, 46-72. 